Glauco Adrieno Westphal ${ }^{1}$, Gerson Costa $^{2}$, Sérgio Gouvêa ${ }^{3}$, Keitiane Michele Kaefer ${ }^{4}$, Rodrigo Soares da Silva $^{4}$, Milton Caldeira Filho ${ }^{1}$

1. Doutor, Médico do Hospital Municipal São José - Joinville (SC), Brasil.

2. Médico do Hospital Municipal São José - Joinville (SC), Brasil.

3. Médico do Hospital Municipal São José - Joinville (SC), Brasil.

4. Médico Residente da Unidade de Terapia Intensiva do Hospital Municipal São José - Joinville (SC), Brasil.

Recebido da Unidade de Terapia Intensiva Geral do Hospital Municipal São José - Joinville (SC), Brasil.

Submetido em 24 de Fevereiro de 2010 Aceito em 08 de Julho de 2010

Autor para correspondência:

Glauco Adrieno Westphal

Rua: Oscar Schneider, 237 - Bairro

Atiradores

CEP: 89203-040 - Joinville (SC), Brasil. Fone: (47) 9964-2295

E-mail: glauco.w@brturbo.com.br

\section{Choque cardiogênico associado à hemorragia subaracnóidea}

\author{
Cardiogenic shock associated with subarachnoid hemorrhage
}

\section{RESUMO}

Complicações sistêmicas são vistas frequentemente em indivíduos acometidos por hemorragia subaracnóidea. Dentre estas alteraçôes podem ocorrer anormalidades eletrocardiográficas que simulam miocardiopatia isquêmica que podem ou não estar associadas com disfunção miocárdica. $\mathrm{O}$ objetivo deste é relatar um caso de associação de hemorragia subaracnóidea com disfunçâo miocárdica e choque cardiogênico. Mulher de 45 anos foi internada com quadro de coma secundário a hemorragia subaracnóidea. À admissão apresentava Glasgow $=7$, Hunt-Hess $=5$ e classificação tomográfica de Fisher $=3$. O aneurisma cerebral de artéria comunicante anterior evidenciado pela arteriografia cerebral foi embolizado com sucesso no segundo dia de internação. Evoluiu com dispnéia e infiltrado pulmonar difuso. Havia alteração da repolarização ventricular em parede lateral, aumento da CK-MB (36 U/L) e hipotensão. $\mathrm{O}$ índice cardíaco de $2,03 \mathrm{~L} / \mathrm{min} / \mathrm{m}^{2}$, a resistência vascular sistêmica 3728 dynes. $\mathrm{seg} / \mathrm{cm}^{5} / \mathrm{m}^{2}$, e a irresponsividade a volume evidenciavam o padrão hemodinâmico de choque cardiogênico. A fração de ejeção do ventrículo esquerdo era de $39 \%$. A cineangiocoronariografia não apresentava lesōes coronarianas obstrutivas. Após 6 dias a paciente foi extubada e ao oitavo dia foi possível a retirada completa da dobutamina. A fraçāo de ejeção passou a $65 \%$. Sucessivos exames de Doppler transcraniano não apresentaram vasoespasmo. A paciente recebeu alta da unidade de terapia intensiva no décimo quarto dia. Pacientes com hemorragia subaracnóidea podem apresentar disfunçấo ventricular e choque cardiogênico, aumentando o risco de isquemia cerebral. $\mathrm{O}$ diagnóstico e a otimização hemodinâmica são essenciais para minimizar os riscos de vasoespasmo e isquemia cerebral.

Descritores: Hemorragia subaracnóidea/etiologia; Choque cardiogênico; Insuficiência cardíaca; Miocardiopatias; Relatos de casos

\section{INTRODUÇÃO}

A hemorragia subaracnóidea (HSA) é uma situação clínica desafiadora, apresentando uma incidência que varia de acordo com o país estudado (2 casos/100.000 habitantes na China a 22,5 casos/100.000 na Finlândia. A faixa etária mais envolvida está entre 40 e 60 anos de idade, podendo afetar desde crianças até idosos. As taxas de mortalidade intra-hospitalar variam entre $33 \%$ e $45 \% .^{(1-3)}$

A HSA está frequentemente associada a complicaçóes sistêmicas como edema pulmonar, distúrbios do sódio e trombose venosa profunda. ${ }^{(2,4)}$ Dentre as alteraçóes cardiovasculares, podem ocorrer anormalidades eletrocardiográficas $(50-90 \%$ dos casos), elevação de enzimas cardíacas, disfunção contrátil global ou segmentar que mimetizam doença cardíaca isquêmica e que podem resultar em choque cardiogêni- 
co. A seguir relatamos um caso de disfunção ventricular grave e choque cardiogênico associado à HSA. . $3,5-7)^{-}$

\section{RELATO DO CASO}

Uma mulher de 45 anos e previamente hígida perdeu a consciência enquanto andava de bicicleta. Foi atendida no local por para-médicos quando apresentava: Glasgow $=7$, pressão arterial (PA) inaudível, pele fria, freqüência cardíaca $(\mathrm{FC})$ $=126$ batimentos por minuto $(\mathrm{bpm})$ e freqüência respiratória $(\mathrm{FR})=10 \mathrm{rpm}$ (respiraçōes por minuto).

Ao dar entrada no serviço de pronto socorro do hospital, apresentava-se com Glasgow $=5, \mathrm{PA}=98 / 66, \mathrm{FC}=70 \mathrm{bpm} \mathrm{e}$ saturação arterial de oxigênio $\left(\mathrm{SaO}_{2}\right)=85 \%$. Após intubação oro-traqueal houve melhora da saturação arterial de oxigênio $\left(\mathrm{SaO}_{2}\right)$, mas apresentava grande quantidade de secreção rósea e espumosa exteriorizada pelo tubo oro-traqueal, quadro que sugeria edema agudo de pulmáo. A radiografia de tórax apresentava infiltrado pulmonar difuso (Figura 1) e o eletrocardiograma, ritmo sinusal, QT longo e alteraçóes da repolarizaçáo em parede lateral (Figura 2). A CK-MB era de $36 \mathrm{U} / \mathrm{L}$ (normal até $16 \mathrm{U} / \mathrm{L}$ ). Havia acidose metabólica com lactato de 2,1 $\mathrm{mmol} / \mathrm{l}$ (normal até $1,6 \mathrm{mmol} / \mathrm{L}$ ).

A tomografia computadorizada de crânio apresentava estiramento difuso de sulcos corticais, velamento hiperdenso de cisternas basais, fissuras silvianas e inter-hemisféricas (classificação tomográfica de Fisher = 3) (Figura 3). A classificação clínica de Hunt-Hess correspondia ao grau 5. Realizada no segundo dia de internação, a arteriografia cerebral evidenciou formação aneurismática sacular bilobulada em complexo comunicante anterior medindo cerca de 5x4 mm (Figura 4).

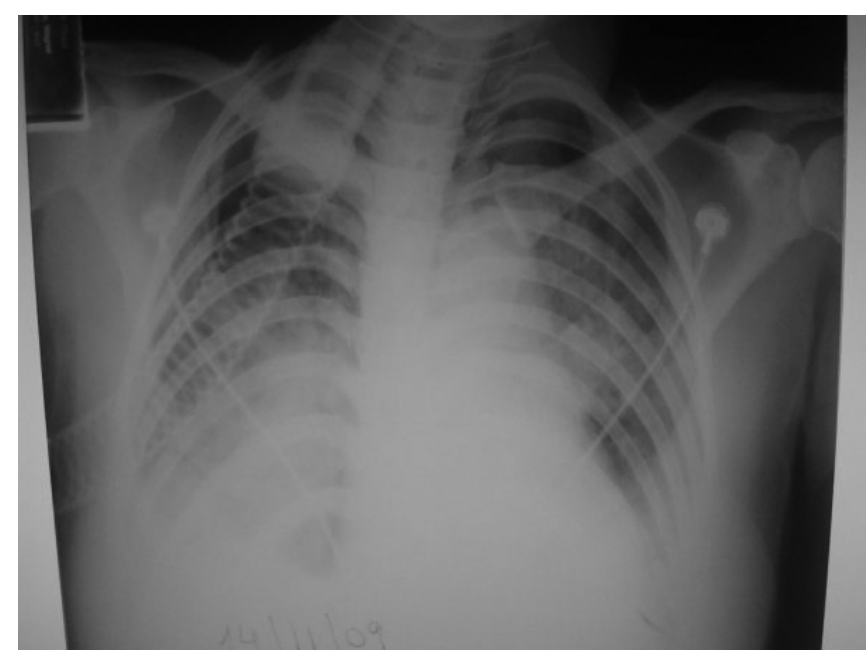

Figura 1 - Radiografia de tórax evidenciando infiltrado pulmonar difuso.
Durante o exame apresentou vasoespasmo mecânico, com melhora após administração de nimodipina intra-arterial. Foi realizada a embolização com micromolas (espirais) de platina de 4, 3 e $2 \mathrm{~mm}$. Após o procedimento endovascular e melhora do nível de consciência, a paciente foi extubada e encaminhada à unidade de terapia intensiva (UTI). Dispnéi$\mathrm{ca}$, necessitou de suporte ventilatório não invasivo (VNI). As

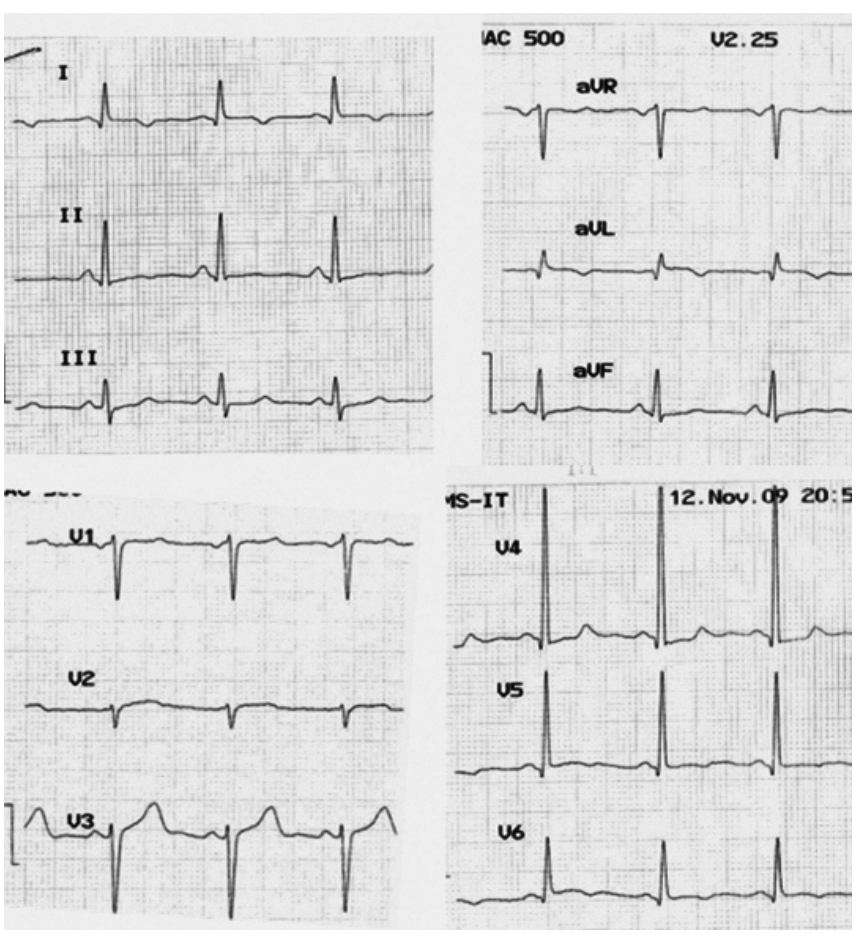

Figura 2 - Eletrocardiograma realizado à admissão da paciente. Observa-se ritmo sinusal, inversáo de onda $T$ nas derivaçóes DI e aVL além de aumento do intervalo QT.

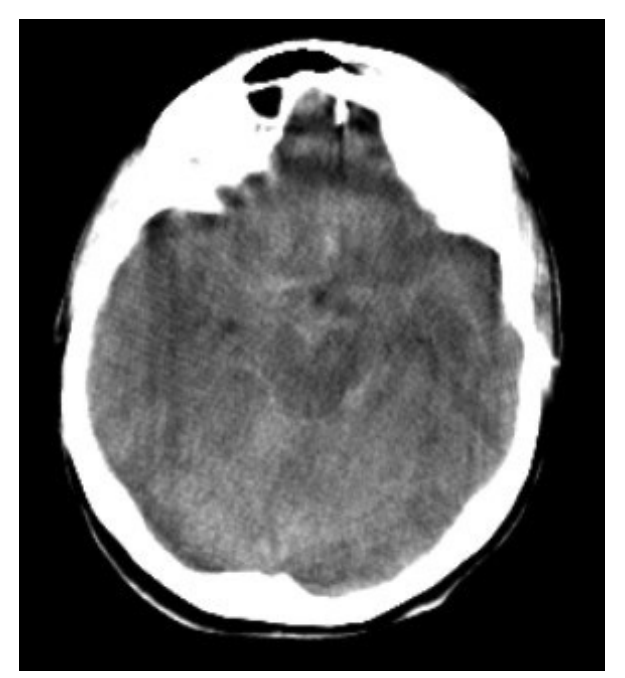

Figura 3 - Imagem axial de tomografia computadorizada evidenciando hemorragia subaracnóide - Fischer III. 


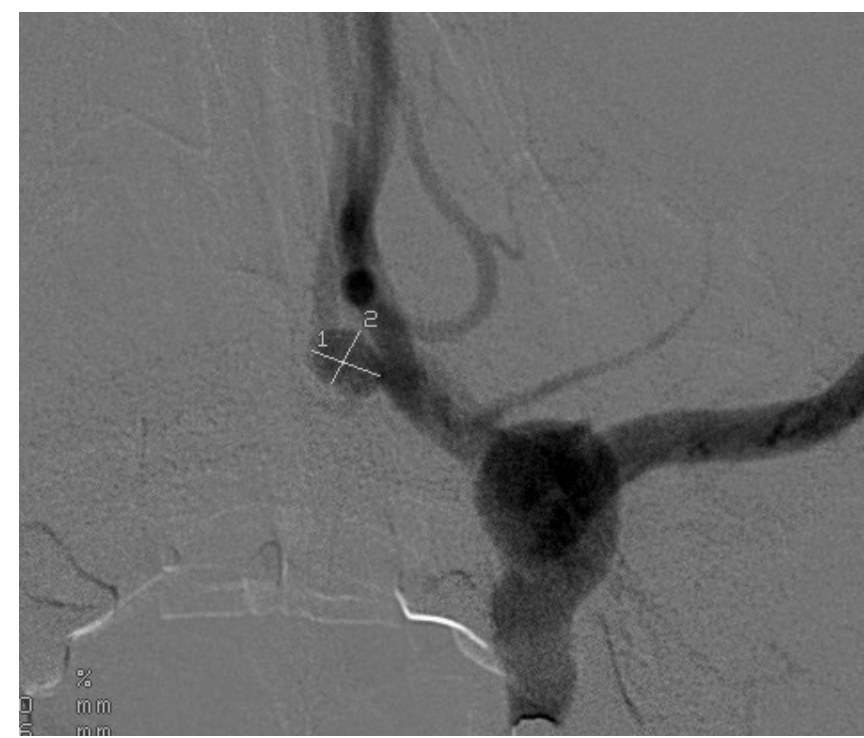

Figura 4 - Angiografia digital evidenciando aneurisma sacular em artéria comunicante anterior.

alteraçôes do eletrocardiograma (ECG) estavam mantidas e o ecocardiograma transtorácico mostrou acinesia e hipocinesia de parede látero-dorsal.

No terceiro dia apresentava-se com Glasgow 15 e sem déficits focais. Houve piora do padrão ventilatório, sendo necessária a intubação oro-traqueal e ventilação mecânica controlada. Evoluiu com hipotensão (80/40) refratária a volume. Iniciada infusão endovenosa de noradrenalina. Avaliação pelo Doppler transcraniano, realizada diariamante, não revelava sinais de vasoespasmo ou repercussóes significativas nas velocidades de fluxo cerebral, mesmo no período de hipotensão arterial.

A variação respiratória da pressão de pulso arterial $(\Delta \mathrm{Pp}=$ $7 \%)$ revelava que a paciente náo era responsiva a volume. $\mathrm{O}$ baixo índice cardíaco $\left(2,03 \mathrm{~L} / \mathrm{min} / \mathrm{m}^{2}\right)$ e a elevada resistência vascular sistêmica $\left(3728\right.$ dynes.seg $/ \mathrm{cm}^{5} / \mathrm{m}^{2}$ ) evidenciavam o padrão hemodinâmico de choque cardiogênico. Novo ecocardiograma transtorácico apresentava acinesia látero-dorsal, intenso comprometimento da funçáo do ventrículo esquerdo, fração de ejeção (FE) de 39\% e sinais de congestão venosa. Houve evidente melhora dos parâmetros hemodinâmicos após iniciar infusão de dobutamina e desmame gradual da noradrenalina.

O cateterismo cardíaco realizado no quinto dia de internação apresentava coronárias sem lesóes obstrutivas, ventrículo esquerdo com hipocinesia moderada a grave e aorta torácica sem alteraçóes (Figura 5).

No sexto dia de internaçáo a paciente foi extubada. Apresentava Glasgow de 15 e o Doppler transcraniano náo evidenciou vasoespasmo. No oitavo dia de internação o novo

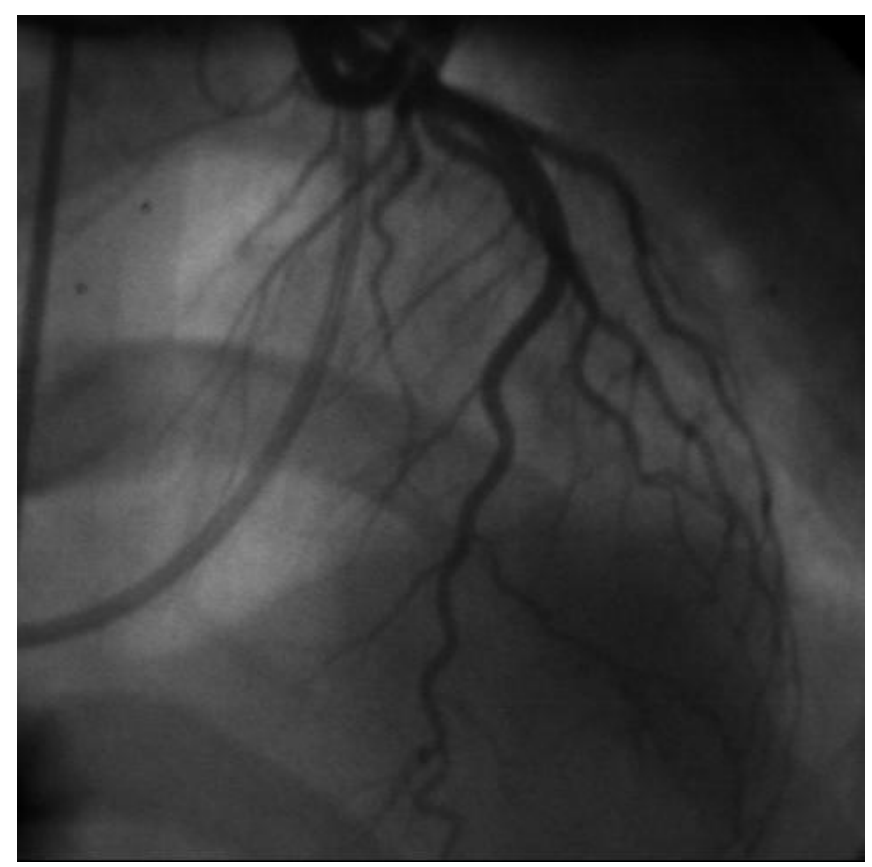

Figura 5 - Coronariografia esquerda sem apresentar lesóes obstrutivas.

ecocardiograma apresentava FE de 65\%, hipocinesia difusa de leve a moderada. Procedeu-se desmame completo da dobutamina.

No décimo primeiro dia houve diminuição no nível de consciência (Glasgow 13). A tomografia computadorizada (TC) de crânio mostrava hidrocefalia discreta, sem sangramento, sem desvio de linha média. Não havia vasoespasmo ao Doppler transcraniano e a conduta foi conservadora.

Com a completa melhora do quadro neurológico a paciente recebeu alta da UTI no décimo quarto dia.

\section{DISCUSSÁO}

Alteraçóes cardíacas, de uma forma geral, afetam cerca de 39\% a 63\% dos pacientes com HSA. Parte dos pacientes apresenta quadros mais graves, sendo que a fisiopatologia da lesão cardíaca permanece mal definida.

A realização de autópsias de indivíduos com HSA demonstrou que não há correlação entre alteração eletrocardiográficas associadas à HSA e doença coronariana ou dano miocárdico de maior relevância. Além disso, estudos posteriores concluíram que tais anormalidades de função ventricular são geralmente reversíveis, sugerindo uma forma de "miocárdio hibernante" não isquêmico. ${ }^{(7)}$ Algumas hipóteses foram delineadas para justificar a disfunção cardíaca associada à HSA, como trombose coronariana, espasmo coronariano e desbalanço entre oferta e consumo de oxigênio no miocárdio se- 
cundário à hipertensão e taquicardia. Acredita-se ainda que a lesão miocárdica pós HSA seja decorrente da combinaçáo de mecanismos neural e humoral. A intensidade de liberação de catecolaminas está fortemente associada a complicações cardíacas em pacientes com HSA. O aspecto histopatológico das lesões cardíacas também não segue um padrão isquêmico, e se associa a sítios de terminação nervosa cardíaca simpática. A liberação excessiva de noradrenalina a partir destas terminaçôes nervosas resulta em constricção vascular seguida de necrose subendocárdica. ${ }^{(6)}$

O diagnóstico da disfunção cardiovascular associada à HSA é baseado em alteraçóes hemodinâmicas, eletrocardiografia, ecocardiografia e dosagem de biomarcadores cardíacos.

As anormalidades eletrocardiográficas associadas à HSA incluem arritmias cardíacas, elevação ou depressão do segmento ST, inversão da onda T, ondas Q patológicas e alteraçóes do intervalo QT. Tais alterações não têm valor prognóstico definido e estáo presentes em grande parte dos pacientes. ${ }^{(6,7)}$

A elevação de biomarcadores cardíacos como CK-MB e troponina I tem sido observada em cerca de $40 \%$ dos pacientes com HSA. Tais alterações frequentemente estão relacionadas a alteraçóes ecocardiográficas, maior risco de complicaçóes cardiovasculares, isquemia cerebral tardia e pior prognóstico.

Anormalidades da função miocárdica são frequentemente $(10 \%$ e $31 \%)$ observadas à ecocardiografia em pacientes com HSA. Estas alterações de contração podem ser globais ou segmentares sem concomitância de doença coronariana confirmada por necropsia ou cineangiocoronariografia.

Nossa paciente apresentou quadro clínico grave de disfunçáo ventricular e choque cardiogênico associados aos padrōes eletrocardiográficos, enzimáticos, ecocardiográficos e angiográficos descritos previamente. A gravidade da HSA, caracterizada pelos achados clínicos (Hunt-Hess $=5$ ) e tomográficos (Fisher $=3$ ), era per se, situação de alto risco para desenvolvimento de vasoespasmo e isquemia cerebral. ${ }^{(1)} \mathrm{A}$ associação do baixo débito cardíaco aumentou sobremaneira este risco de hipofluxo e isquemia cerebral. ${ }^{(1,8)}$ Possivelmente a terapia de suporte hemodinâmico, associada a monitorização de fluxo sanguíneo cerebral pelo Doppler transcraniano, contribuiu com a não ocorrência de isquemia cerebral.
Concluindo, em pacientes com HSA pode haver disfunção ventricular e a ocorrência de grave acometimento de fluxo sanguíneo global, comprometimento do fluxo sanguíneo cerebral e aumento do risco de isquemia cerebral. Portanto, deve-se atentar precocemente para esta possibilidade diagnóstica e para a necessidade da otimização hemodinâmica, buscando minimizar a ocorrência de vasoespasmo e os riscos de isquemia.

\section{ABSTRACT}

Systemic complications are frequent in subarachnoid hemorrhage patients. Among these complications, electrocardiographic abnormalities simulating ischemic cardiomyopathy may occur, possibly associated with myocardial dysfunction. This manuscript aims to report a case of subarachnoid hemorrhage associated with myocardial dysfunction and cardiogenic shock. A 45 years old woman was admitted with subarachnoid hemorrhage and coma, showing Glasgow scale $=7$, Hunt-Hess $=5$ and Fischer computed tomography classification $=3$. On the second day, the patient underwent anterior cerebral communicant artery aneurysm embolization. The clinical evaluation revealed diffuse pulmonary infiltration, dyspnea and hypotension. Additional tests showed electrocardiographic lateral wall repolarization changes and elevated creatine kinase-MB fraction $(36 \mathrm{U} / \mathrm{L})$. The cardiac index was $2.03 \mathrm{~L} /$ minute $/ \mathrm{m}^{2}$, Vascular systemic resistance was 3728 dynes.sec/ $\mathrm{cm}^{2}$. The non-responsiveness to volume demonstrated a cardiogenic shock pattern. The ventricular ejection fraction was 39\%. The coronariography was normal, showing no obstructive lesions. Six days later the patient was removed from respiratory support and after eight days the dobutamine infusion was discontinued. The ejection fraction recovered up to $65 \%$. Serial transcranial Doppler evaluations did not show vascular spasm. After ten days the patient was discharged from the intensive care unit. Patients with subarachnoid hemorrhage may be complicated with ventricular dysfunction and cardiogenic shock, increasing the cerebral ischemia risk. Diagnosis optimization and hemodynamic stabilization are essential to minimize the risk of cerebral vasospasm and ischemia.

Keywords: Subarachnoid hemorrhage/etiology; Shock, cardiogenic; Heart failure; Cardiomyopathies; Case reports

\section{REFERÊNCIAS}

1. Bederson JB, Connolly ES Jr, Batjer HH, Dacey RG, Dion JE, Diringer MN, Duldner JE Jr, Harbaugh RE, Patel AB, Rosenwasser RH; American Heart Association. Guidelines for the management of aneurysmal subarachnoid hemorrhage: a statement for healthcare professionals from a special writing group of the Stroke Council, American Heart Association. Stroke. 2009;40(3):994-1025. Erratum in: Stroke. 2009;40(7):e518.

2. Schievink WI. Intracranial aneurysms. N Engl J Med. 1997;336(1):28-40. Review. Erratum in: N Engl J Med. 
1997;336(17):1267.

3. Kothavale A, Banki NM, Kopelnik A, Yarlagadda S, Lawton MY, Ko N, et al. Predictors of left ventricular regional wall motion abnormalities after subarachnoid hemorrhage. Neurocrit Care. 2006;4(3):199-205.

4. Suarez JI, Tarr RW, Selman WR. Aneurysmal subarachnoid hemorrhage. N Engl J Med. 2006;354(4):387-96.

5. Lee HC, Yen HW, Lu YH, Lee KT, Voon WC, Lai WT, Sheu SHS. A case of subarachnoid hemorrhage with persistent shock and transient ST elevation simulating acute myocardial infarction. Kaohsiung J Med Sci.
2004;20(9):452-6.

6. Letsas KP, Gavrielatos G, Sideris A. Neurocardiogenic injury in subarachnoid hemorrhage. J Emerg Med. 2008;35(3):315-6.

7. Stevens RD, Nyquist PA. The systemic implications of aneurismal subarachnoid hemorrhage. J Neurol Sci. 2007;261(1-2):143-56.

8. Mayer SA, Lin J, Homma S, Solomon RA, Lennihan L, Sherman D, et al. Myocardial injury and left ventricular performance after subarachnoid hemorrhage. Stroke. 1999;30(4):780-6. 